\title{
Verification and Validation of a Three-Dimensional Composite Impact Model With Tabulated Input
}

\author{
Robert K. Goldberg ${ }^{1}$ and Trenton M. Ricks ${ }^{2}$ \\ NASA Glenn Research Center, Cleveland, Ohio \\ Kelly S. Carney ${ }^{3}$ and Paul DuBois ${ }^{4}$ \\ George Mason University, Fairfax, Virginia \\ Bilal Khaled $^{5}$, Loukham Shyamsunder ${ }^{6}$ and Subramaniam Rajan ${ }^{7}$ \\ Arizona State University, Tempe, Arizona \\ and \\ Gunther Blankenhorn ${ }^{8}$ \\ Livermore Software Technology Corporation, Livermore, California
}

\begin{abstract}
A material model which incorporates several key capabilities which have been identified by the aerospace community as lacking in the composite impact models currently available in the commercial transient dynamic finite element code LS-DYNA ${ }^{\circledR}$ has been developed. The material model utilizes experimentally based tabulated input to define the evolution of plasticity and damage as opposed to specifying discrete input parameters (such as modulus and strength. The plasticity portion of the orthotropic, three-dimensional, macroscopic composite constitutive model is based on an extension of the Tsai-Wu composite failure model into a generalized yield function with a non-associative flow rule. The capability to account for the rate and temperature dependent deformation response of composites has also been incorporated into the material model. For the damage model, a strain equivalent formulation is utilized to allow for the uncoupling of the deformation and damage analyses. In the damage model, a diagonal damage tensor is defined to account for the directionally dependent variation of damage. However, the terms in the damage matrix are semi-coupled such that the damage in a particular coordinate direction is a function of the stresses and plastic strains in all of the coordinate directions. For the failure model, a tabulated approach is utilized in which a stress or strain based invariant is defined as a function of the location of the current stress state in stress space to define the initiation of failure, which allows an arbitrarily shaped failure surface to be defined. A systematic series of validation and verification studies, at a variety of length scales ranging from single element simulations to simulations of a flat panel impact test, have been performed to fully exercise and evaluate the capabilities of the developed model.
\end{abstract}

\section{Introduction}

As composite materials are gaining increased use in aircraft components where impact resistance is critical (such as the turbine engine fan case), the need for accurate material models to simulate the deformation, damage and failure

\footnotetext{
${ }^{1}$ Research Aerospace Engineer, Materials and Structures Division, AIAA Associate Fellow.

${ }^{2}$ Aerospace Engineer, Materials and Structures Division, AIAA Member.

${ }^{3}$ Research Scientist, Center for Collision Safety and Analysis.

${ }^{4}$ Research Scientist, Center for Collision Safety and Analysis.

${ }^{5}$ Graduate Student, School of Sustainable Engineering and the Built Environment.

${ }^{6}$ Graduate Student, School of Sustainable Engineering and the Built Environment.

${ }^{7}$ Professor, School of Sustainable Engineering and the Built Environment.

${ }^{8}$ Software Engineer.
} 
response of polymer matrix composites under impact conditions is gaining in importance. While there are several material models currently available within commercial transient dynamic codes such as LS-DYNA® (1) to analyze the impact response of composites, areas have been identified where the predictive capabilities of these models can be improved. Most importantly, the existing models often require extensive correlation based on structural level impact tests, which significantly limits the ability to use these models as predictive tools. Furthermore, most of the existing models apply either a plasticity based approach (such as that used by Sun and Chen (2)) or a continuum damage mechanics approach (such as that used by Matzenmiller et al (3)) to simulate the nonlinearity that takes place in the composite response. As documented in detail by Goldberg, et al $(4,5)$, either of these approaches can capture certain aspects of the actual composite behavior. However, optimally, combining a plasticity based deformation model (to capture the rate dependence and significant nonlinearity, particularly in shear, observed in the composite response) with a damage model (to account for the changes in the unloading modulus observed as the material is unloaded from various stress levels, as well as to account for strain softening observed after the peak stress is reached) can provide advantages over using one approach or the other. In addition, the input to current material models generally consists of point-wise properties (such as the modulus, failure stress or failure strain in a particular coordinate direction) that leads to curve fit approximations to the material stress-strain curves. This type of approach either leads to models with only a few parameters, which provide a crude approximation at best to the actual material response, or to models with many parameters which require a large number of complex tests to characterize. An improved approach is to use tabulated data, obtained from a well-defined set of straightforward experiments. Using tabulated data allows the actual material response data to be entered in discretized form, which permits a more accurate representation of the actual material response.

To begin to address these needs, a new composite material model is being developed and implemented for use within LS-DYNA®. The material model is being implemented as MAT 213. The material model is meant to be a fully generalized model suitable for use with any composite architecture (laminated or textile). The deformation model is based on extending the commonly used Tsai-Wu composite failure model (6) to a strain hardening plasticity model with a non-associative flow rule. For the damage model, a strain equivalent formulation is used in which the deformation and damage calculations can be uncoupled. A significant feature in the developed damage model is that a semi-coupled approach has been utilized in which a load in a particular coordinate direction results in damage (and thus stiffness reduction) in multiple coordinate directions. While different from the approach used in many existing damage mechanics models (3) in which a load in a particular coordinate direction only leads to a stiffness reduction in the load direction, this approach has the potential to more accurately reflect the damage behavior that actually takes place, particularly for composites with more complex fiber architectures. For the failure model implemented within the developed methodology, a tabulated approach has been developed (7), in which the actual experimental threedimensional failure envelope for a composite is entered in a tabulated fashion. Specifically, a stress or strain based invariant leading to the initiation of failure is defined as a function of the location of the current stress state in stress space. In this manner, an arbitrary failure surface can be easily defined based on actual experimental data in combination with numerical data obtained using any desired existing failure model. By this approach, the failure surface is not limited to an arbitrary shape, but can more accurately represent the actual failure response of the composite.

In the following sections of this abstract, a brief summary of the plasticity-based deformation model is presented. The key aspects of the damage model and its characterization are then described. The failure model is also summarized. Since the initial development cycle for MAT 213 has been completed, a rigorous program to thoroughly exercise and validate the material model across a variety of length scales is currently being carried out. Details of the verification study will be outlined in the abstract, with the expectation that more complete discussions of the verification studies will be presented in the final paper

\section{Deformation Model Overview}

A complete description of the deformation model is given in Goldberg et al $(4,5)$. A summary of the key features of the model is presented here. In the deformation model, a general quadratic three-dimensional orthotropic yield function based on the Tsai-Wu failure model is specified as follows, where 1, 2, and 3 refer to the principal material directions: 


$$
\begin{aligned}
f(\sigma)=-1+\left(\begin{array}{llllll}
F_{1} & F_{2} & F_{3} & 0 & 0 & 0
\end{array}\right)\left(\begin{array}{l}
\sigma_{11} \\
\sigma_{22} \\
\sigma_{33} \\
\sigma_{12} \\
\sigma_{23} \\
\sigma_{31}
\end{array}\right) \\
+\left(\begin{array}{llllllll}
\sigma_{11} & \sigma_{22} & \sigma_{33} & \sigma_{12} & \sigma_{23} & \sigma_{31}
\end{array}\right)\left(\begin{array}{cccccc}
F_{11} & F_{12} & F_{13} & 0 & 0 & 0 \\
F_{12} & F_{22} & F_{23} & 0 & 0 & 0 \\
F_{13} & F_{23} & F_{33} & 0 & 0 & 0 \\
0 & 0 & 0 & F_{44} & 0 & 0 \\
0 & 0 & 0 & 0 & F_{55} & 0 \\
0 & 0 & 0 & 0 & 0 & F_{66}
\end{array}\right)\left(\begin{array}{l}
\sigma_{11} \\
\sigma_{22} \\
\sigma_{33} \\
\sigma_{12} \\
\sigma_{23} \\
\sigma_{31}
\end{array}\right)
\end{aligned}
$$

In the yield function, $\sigma \mathrm{ij}$ represents the stresses and Fij and Fk are coefficients that evolve and are functions of the current values of the stresses in the various coordinate directions. By allowing the coefficients to vary, the yield surface evolution and hardening in each of the material directions can be precisely defined. The values of the normal and shear coefficients can be determined by simplifying the yield function for the case of unidirectional tensile and compressive loading in each of the coordinate directions along with shear tests in each of the shear directions. In the above equation, the stresses are the current value of the yield stresses in the normal and shear directions. To determine the values of the off-axis coefficients (which are required to capture the stress interaction effects), the results from $45^{\circ}$ off-axis tests (an arbitrarily chosen angle) in the various coordinate directions can be used. The values of the offdiagonal terms in the yield function can also be modified as required in order to ensure that the yield surface is convex (5).

A non-associative flow rule is used to compute the evolution of the components of plastic strain. The plastic potential for the flow rule is shown in Equation (2) below:

$$
h=\sqrt{H_{11} \sigma_{11}^{2}+H_{22} \sigma_{22}^{2}+H_{33} \sigma_{33}^{2}+2 H_{12} \sigma_{11} \sigma_{22}+2 H_{23} \sigma_{22} \sigma_{33}+2 H_{31} \sigma_{33} \sigma_{11}+H_{44} \sigma_{12}^{2}+H_{55} \sigma_{23}^{2}+H_{66} \sigma_{31}^{2}}
$$

where $\sigma \mathrm{ij}$ are the current values of the stresses and Hij are independent coefficients, which are assumed to remain constant. The values of the coefficients are computed based on average plastic Poisson's ratios [4, 5]. The plastic potential function in Equation (2) is used in a flow law to compute the components of the plastic strain rate, where the usual normality hypothesis from classical plasticity (8) is assumed to apply.

Given the flow law, the principal of the equivalence of plastic work (8) can be used to determine that the plastic potential function, $\mathrm{h}$, can be defined as the effective stress and the plastic multiplier can be defined as the effective plastic strain rate.

To compute the current value of the yield stresses needed for the yield function, tabulated stress-strain curves are used to track the yield stress evolution. The user is required to input twelve stress versus plastic strain curves. Specifically, the required curves include uniaxial tension curves in each of the normal directions $(1,2,3)$, uniaxial compression curves in each of the normal directions $(1,2,3)$, shear stress-strain curves in each of the shear directions (1-2, 2-3 and 3-1), and 45 degree off-axis tension or compression curves in each of the 1-2, 2-3 and 3-1 planes. The 45 degree curves are required in order to properly capture the stress interaction effects. By utilizing tabulated stressstrain curves to track the evolution of the deformation response, the experimental stress-strain response of the material can be captured exactly without any curve fit approximations. The required stress-strain data can be obtained either from actual experimental test results or by appropriate numerical experiments utilizing stand-alone codes. The ability to account for rate and temperature effects has also been incorporated into the deformation model. To track the evolution of the deformation response along each of the stress-strain curves, the effective plastic strain is chosen to be the tracking parameter. Using a numerical procedure based on the radial return method (8) in combination with an iterative approach, the effective plastic strain is computed for each time/load step. The stresses for each of the tabulated input curves corresponding to the current value of the effective plastic strain are then used to compute the yield function coefficients. 


\section{Damage Model Overview}

The deformation portion of the material model provides the majority of the capability of the model to simulate the nonlinear stress-strain response of the composite. However, in order to capture the changes in the unloading modulus observed as the material is unloaded from various stress levels and the local softening of the stress-strain response that is often observed in composites (9), a complementary damage law is required. Strain equivalence is assumed in the damage law formulation, therefore the total, elastic and plastic strains in the actual and effective stress spaces are the same for every time step (10). The utilization of strain equivalence permits the plasticity and damage calculations to be uncoupled, as all of the plasticity computations can take place in the effective stress space.

The first step in the development of the damage model is to relate the actual stresses to a set of effective stresses by use of a damage tensor M. Given the usual assumption that the actual stress tensor and the effective stress tensor are symmetric, the actual stresses can be related to the effective stresses in the following manner, where the damage tensor $\mathbf{M}$ is assumed to have a maximum of 36 independent components:

$$
\left(\begin{array}{l}
\sigma_{11} \\
\sigma_{22} \\
\sigma_{33} \\
\sigma_{12} \\
\sigma_{23} \\
\sigma_{13}
\end{array}\right)=[\mathbf{M}]\left(\begin{array}{l}
\sigma_{11}^{e f f} \\
\sigma_{22}^{\text {eff }} \\
\sigma_{33}^{\text {eff }} \\
\sigma_{12}^{\text {eff }} \\
\sigma_{23}^{\text {eff }} \\
\sigma_{13}^{\text {eff }}
\end{array}\right)
$$

In order to maintain a one to one relationship between the effective stresses and the actual stresses (i.e. to ensure that a uniaxial load in the actual stress space does not result in a multi-axial load in the effective stress space), the damage tensor is assumed to be diagonal An implication of a diagonal damage tensor is that loading the composite in a particular coordinate direction only leads to a stiffness reduction in the direction of the load due to the formation of matrix cracks perpendicular to the direction of the load. However, as discussed in detail in Goldberg, et al. (5), in actual composites, particularly those with complex fiber architectures, a load in one coordinate direction can lead to stiffness reductions in multiple coordinate directions. To maintain a diagonal damage tensor while still allowing for the damage interaction in at least a semi-coupled sense, each term in the diagonal damage matrix should be a function of the plastic strains in each of the normal and shear coordinate directions, as follows for the example of the $\mathrm{M}_{11}$ term for the plane stress case:

$$
M_{11}=M_{11}\left(\varepsilon_{11}^{p}, \varepsilon_{22}^{p}, \varepsilon_{12}^{p}\right)
$$

Note that plastic strains are chosen as the "tracking parameter" due to the fact that, within the context of the developed formulation, the material nonlinearity during loading is simulated by use of a plasticity based model. The plastic strains therefore track the current state of load and deformation in the material. To explain this concept of damage coupling further, assume a load is applied in the 1 direction to an undamaged specimen. The undamaged modulus in the 1-direction is $E_{11}$ and the undamaged modulus in the 2-direction is $E_{22}$. The stress-strain response of the material is assumed to become nonlinear (represented in the current model by the accumulation of plastic strain) and damage is assumed to occur. The original specimen is unloaded and reloaded elastically in the 1-direction. Due to the damage, the reloaded specimen has a reduced modulus in the 1-direction of $E_{11}^{d 11}$. The reduced area and modulus are a function of the damage induced by the loading and resulting nonlinear deformation in the 1 direction (reflected as plastic strain) as follows:

$$
E_{11}^{d 11}=\left(1-d_{11}^{11}\left(\varepsilon_{11}^{p}\right)\right) E_{11}
$$

where $d_{11}^{11}$ is the damage in the 1-direction due to a load in the 1-direction. Note that damage is only assumed to initiate once plastic strains have started to accumulate. Alternatively, if the damaged specimen was reloaded elastically in the 2-direction, due to the assumed damage coupling resulting from the load in the 1-direction, the 
reloaded specimen would have a reduced modulus in the 2-direction of $E_{22}^{d 11}$. The reduced modulus is also a function of the damage induced by the load and resulting nonlinear deformation in the 1-direction as follows:

$$
E_{22}^{d 11}=\left(1-d_{11}^{22}\left(\varepsilon_{11}^{p}\right)\right) E_{22}
$$

where $d_{11}^{22}$ is the damage in the 2-direction due to a load in the 1-direction. Similar arguments can be made and equations developed for the situation where the original specimen is loaded in the 2-direction.

For the case of multiaxial loading, the semi-coupled formulation needs to account for the fact that as the load is applied in a particular coordinate direction, the loads are acting on damaged areas due to the loads in the other coordinate directions, and the load in a particular direction is just adding to the damaged area. For example, if one loaded the material in the 2-direction first, the reduced modulus in the 1-direction would be equal to $E_{11}^{d 22}$. If one would then subsequently load the material in the 1-direction, the baseline modulus in the 1-direction would not be equal to the

original modulus $E_{11}$, but instead the reduced modulus $E_{11}^{d 22}$. These results suggest that the relation between the actual stress and the effective stress should be based on a multiplicative combination of the damage terms as opposed to an additive combination of the damage terms. For example, for the case of plane stress, the relation between the actual and effective stresses could be expressed as follows:

$$
\begin{aligned}
& \sigma_{11}=\left(1-d_{11}^{11}\right)\left(1-d_{22}^{11}\right)\left(1-d_{12}^{11}\right) \sigma_{11}^{e f f} \\
& \sigma_{22}=\left(1-d_{11}^{22}\right)\left(1-d_{22}^{22}\right)\left(1-d_{12}^{22}\right) \sigma_{22}^{e f f} \\
& \sigma_{12}=\left(1-d_{11}^{12}\right)\left(1-d_{22}^{12}\right)\left(1-d_{12}^{12}\right) \sigma_{12}^{e f f}
\end{aligned}
$$

where for each of the damage terms the subscript indicates the direction of the load which initiates the particular increment of damage and the superscript indicates the direction in which the damage takes place. For the full threedimensional case the actual stresses would be functions of damage parameters in all six coordinate directions.

To properly characterize the damage model, an extensive set of test data is required. Due to the tabulated nature of the input, each of the damage parameters $\left(d_{11}^{22}, d_{11}^{22}\right.$, etc.) has to be determined as a function of the plastic strain in a particular coordinate direction (such as $\varepsilon_{11}^{p}$ ). For example, to determine the damage terms for the case of loading in the 1-direction, a composite specimen has to be loaded to a certain plastic strain level in the 1-direction. The material is then unloaded to a state of zero stress, and then reloaded elastically in each of the coordinate directions to determine the reduced modulus of the material in each of the coordinate directions. From this data, the required damage parameters can be determined.

\section{Failure Model Overview}

The majority of available composite failure models utilize mathematical functions to describe the failure surface, which impose a specific shape on the failure surface. For example, for the commonly used Tsai-Wu failure criteria (6), the failure surface is elliptical (in 2D) or ovoid (in 3D) due to the quadratic nature of the equation defining the failure surface. An example of this type of failure surface is shown in Figure 1. In reality, however, the failure surfaces of actual composites often do not exhibit this simple shape. Many actual failure surfaces cannot be easily defined by a mathematical function of the stresses. Alternatively, as shown in Figure 1, one potential method of defining the points in the failure surface is to use a cylindrical type of coordinate system. In this approach, for the inplane stresses, a variable $\theta$ defines the relative location of the point on the failure surface in stress space, while a second variable $r$ defines the "magnitude" of the failure surface point in the stress space location. Since the relationship between " $r$ " and " $\theta$ " also cannot be easily defined by a mathematical function for a realistic composite failure surface, a tabulated approach, where a series of " $r$ " and " $\theta$ " pairs are explicitly defined for a given failure surface, can provide a more accurate representation of the failure surface. The tabulated approach allows for the use of experimentally defined failure surface data, a failure surface defined using any existing failure model, or a combination of experimental and numerically obtained "virtual" data. The combined approach can allow for the case where actual 
failure data are only available for a portion of the total stress space, with "virtual" data being required to fill in the gaps in the failure surface.

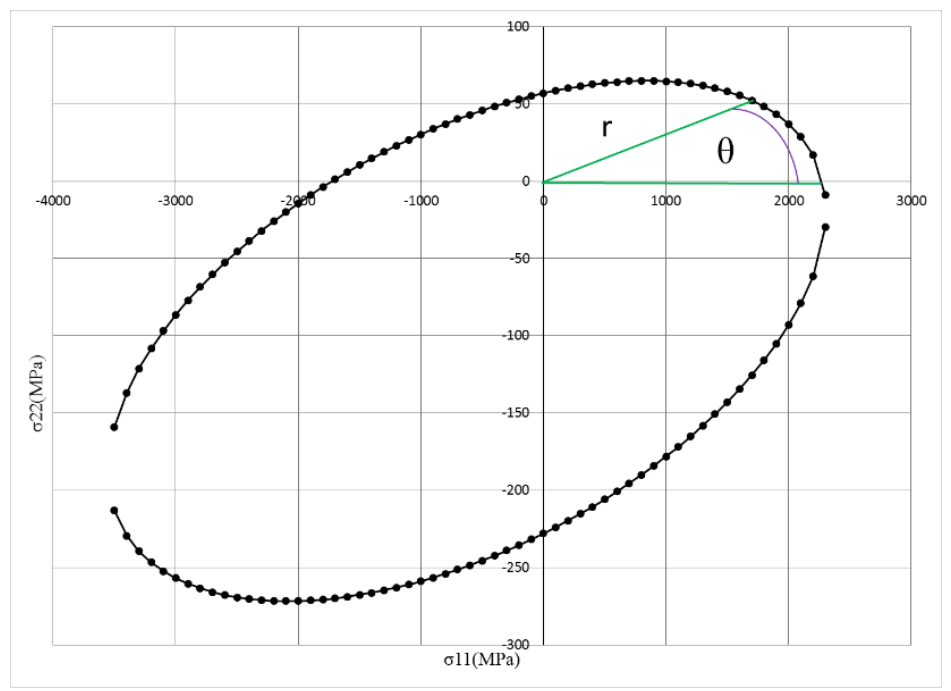

Figure 1: Example failure surface with variable definitions.

For the tabulated failure surface definition proposed in this study, appropriate independent and dependent variables need to be defined. The independent variables need to define the location of a point on the failure surface in stress space, and the dependent variable needs to define the magnitude of the failure surface point along the lines defined by the independent variables. For the current approach, the in-plane and out-of-plane responses are considered separately. The definition of the in-plane failure surface is defined here. The out of plane failure surface is defined in a similar manner and a coupling term is used to combine the effects of the in-plane and out-of-plane failure responses. For the in-plane failure surface definition, two independent variables are defined. The first independent variable will be the ratio of the shear stress to the shear failure stress. For selected values of this shear ratio, the location of each defined point on the failure surface in stress space is specified by defining the angle of the point in the $\sigma_{11}-\sigma_{22}$ plane, as shown in Figure 1, which becomes the second independent variable. Using simple geometric principals, the angle $\theta$ for each defined point on the failure surface can be defined in terms of the stresses $\sigma_{11}$ and $\sigma_{22}$ as shown below. To ensure a set of unique, monotonically increasing angles from $-180^{\circ}$ to $180^{\circ}$, if the stress $\sigma_{22}$ is negative the computed angle is multiplied by -1 as shown below:

$$
\begin{aligned}
& \theta=\cos ^{-1}\left(\frac{\sigma_{11}}{\sqrt{\sigma_{11}^{2}+\sigma_{22}^{2}}}\right) \\
& \theta_{a c t}=-\theta \text { if } \sigma_{22} \leq 0
\end{aligned}
$$

For the dependent variable, which is used to define the magnitude of the failure surface point given a particular location in stress space, a stress invariant first identified by Fleischer et al (11) is used, defined as follows for the plane stress case:

$$
r=\sqrt{\sigma_{11}^{2}+\sigma_{22}^{2}+2 \sigma_{12}^{2}}
$$

This invariant can be considered to be like a "radius" from the origin to the failure surface. The factor of 2 in front of the shear stress term reflects the symmetry of the stress tensor. Stresses or strains can be used in defining the dependent variable, making the model more general. Using an invariant type of term also allows for the stress interactions to be more appropriately accounted for in the failure definition and helps to ensure that the failure definition will be accurate 
for a variety of loading conditions. Plots of the radius versus angle and be generated for a variety of shear stresses, as is shown in Figure 2. This process converts the failure surface to a single valued function, which can be implemented in a tabular fashion. To ensure that a well-behaved function is obtained, the failure stresses in the original failure surface can be scaled and the center of the failure surface can be redefined. More details of this process is discussed in Goldberg, et al (7).

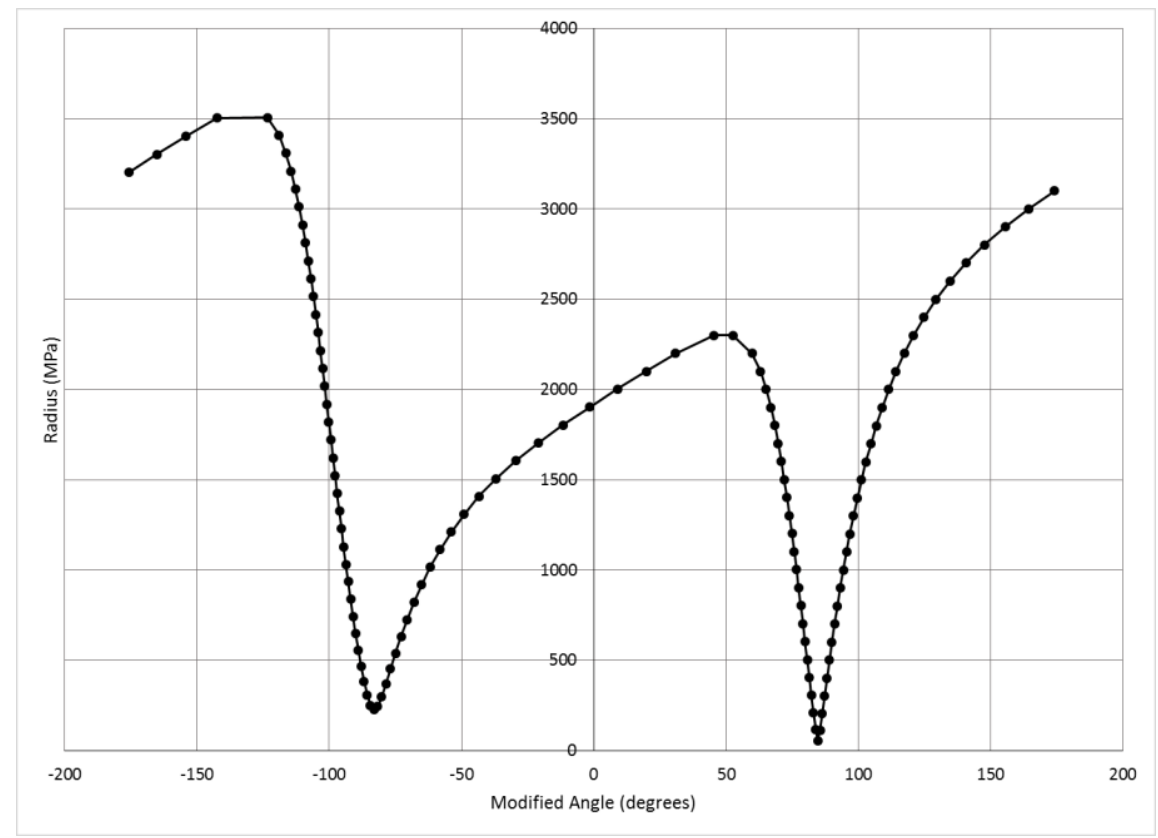

Figure 2. Plot of radius versus angle for example failure surface.

\section{Verification and Validation Studies}

A verification and validation process is currently being carried out to exercise the material model for a number of material system with varying fiber architectures, including unidirectional composites, weaves and tri-axial braids. The goal of this V\&V process is to demonstrate that the underlying theory is correctly implemented into LS-DYNA, as well as to demonstrate that the methodology can accurately capture the physics of an impact event. The overall process is illustrated in Figure 3. As shown in the figure, the first step of the verification process is to conduct a series of single element analyses. In the single element analyses, the loading conditions that were used to provide the twelve input curves for the deformation portion of the material model are simulated, specifically uniaxial tension curves in each of the normal directions $(1,2,3)$, uniaxial compression curves in each of the normal directions $(1,2,3)$, shear stressstrain curves in each of the shear directions (1-2, 2-3 and 3-1), and 45 degree off-axis tension or compression curves in each of the 1-2, 2-3 and 3-1 planes. By examining the results from these analyses, the ability of the code to accurately replicate the input data is being determined. In this way, the analyses can determine that the theory is implemented into the code correctly. By using single elements for this first set of verification and validation studies, complexities of the analysis caused by complicated boundary conditions, element interactions and edge effects can be avoided.

The second step in the verification and validation process is to conduct a set of "coupon" level analyses. In these studies, the actual coupon level tests conducted to obtain the twelve input curves are simulated. The actual coupon geometries and boundary conditions are utilized in the analysis. These simulations examine the ability of the material model to accurately simulate the material response when more realistic boundary conditions and stress gradients are applied to the model, along with the complexities involved in conducting an analysis with multiple elements. However, since these coupon tests were utilized to obtained fundamental material property data, the loading conditions that were applied in the tests were still relatively simple. These simulations then fall somewhere between a verification test and a validation test. Since the goal of these simulations is still to ensure that the overall average stress-strain response obtained from the analyses correlates to the relevant input curves, these simulations are still verification tests. 
However, since more realistic geometries and boundary conditions are applied, these simulations begin to serve the purpose of a validation test, in which the ability of the material model to accurately simulate the material behavior can be determined.

Once the verification tests are completed, the next step of the $\mathrm{V} \& \mathrm{~V}$ process is to carry out a full set of validation studies. The goal of these analyses is to determine if the material model can accurately simulate the physics of a composite impact event. For this study, various flat panel impact tests are being simulated in order to validate the ability of the code to predict the impact response of composite structures under realistic impact loading conditions. Since the material model was specifically designed to more accurately simulate the impact response of composites, the ability of the code to appropriately simulate the response of composite structures subject to realistic impact conditions is critical.

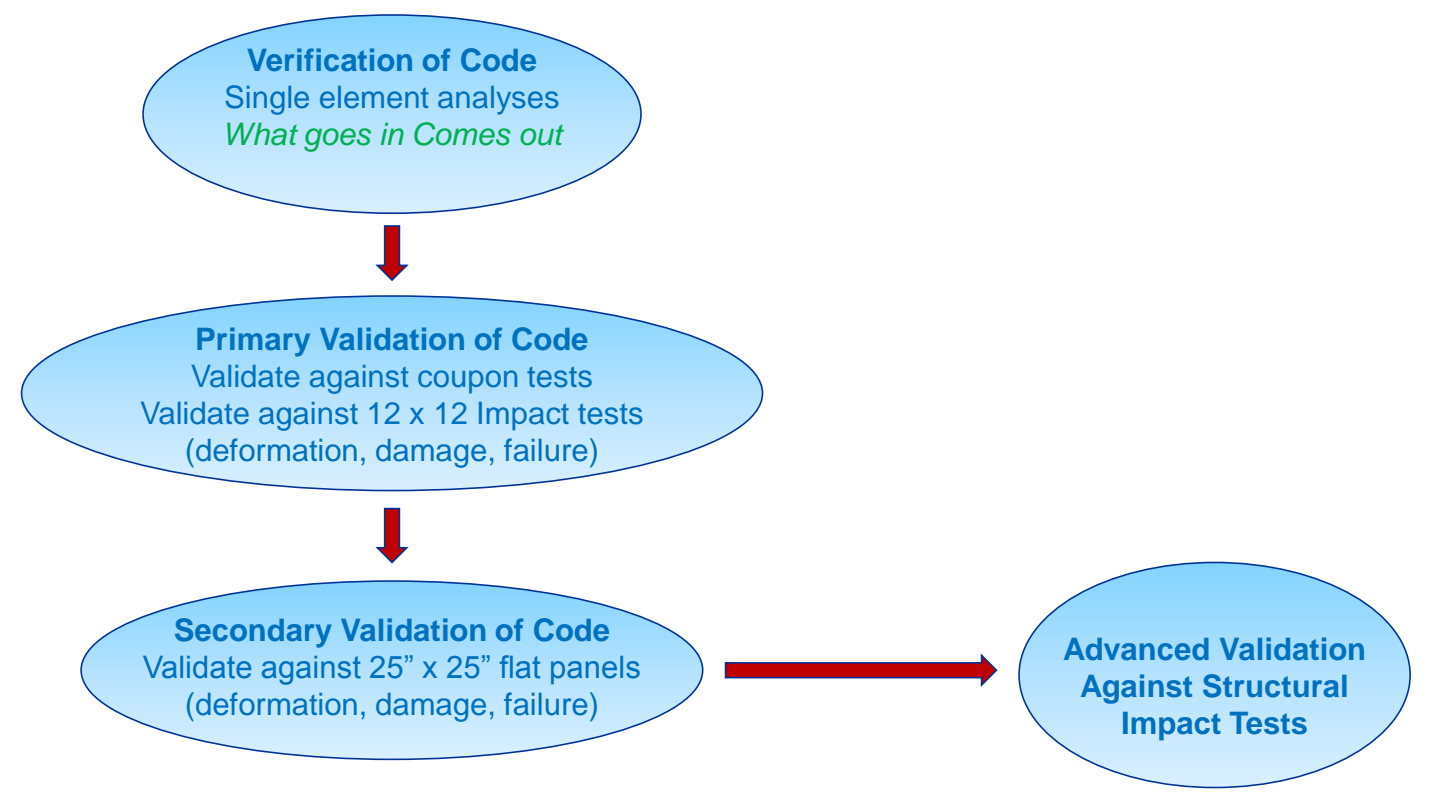

Figure 3. Schematic of the verification and validation process.

As mentioned previously, the verification and validation process is being carried out for a variety of material systems under the current effort. Single element analyses were carried out on a T700/PR520 2D triaxially braided $\left[0^{\circ} / 60^{\circ} /-60^{\circ}\right]$ composite. This material system has $24 \mathrm{~K}$ filaments per tow in the axial $\left(0^{\circ}\right)$ direction, $12 \mathrm{~K}$ filaments per tow in the bias/braider $\left(+/-60^{\circ}\right.$ with respect to the axial tows) directions, and six layers of triaxially braided plies through the thickness. The finite element model used to carry out these simulations for the cases of normal loading (longitudinal tension/compression, in-plane transverse tension/compression, out-of-plane tension/compression) is shown in Figure 4. In these models, the fiber direction is parallel to the global X-direction. The nodes of the unit cube on the bottom side are constrained against motion normal to the X-Z plane; the other two degrees-of-freedom are also constrained at one of those four nodes to preclude rigid body translation. Prescribed pressure is applied to the opposite face providing for a state of uniaxial stress. While the fiber direction remains constant, the nodal constraints and pressure loading change to simulate uniaxial stress in the other two orthogonal directions. 

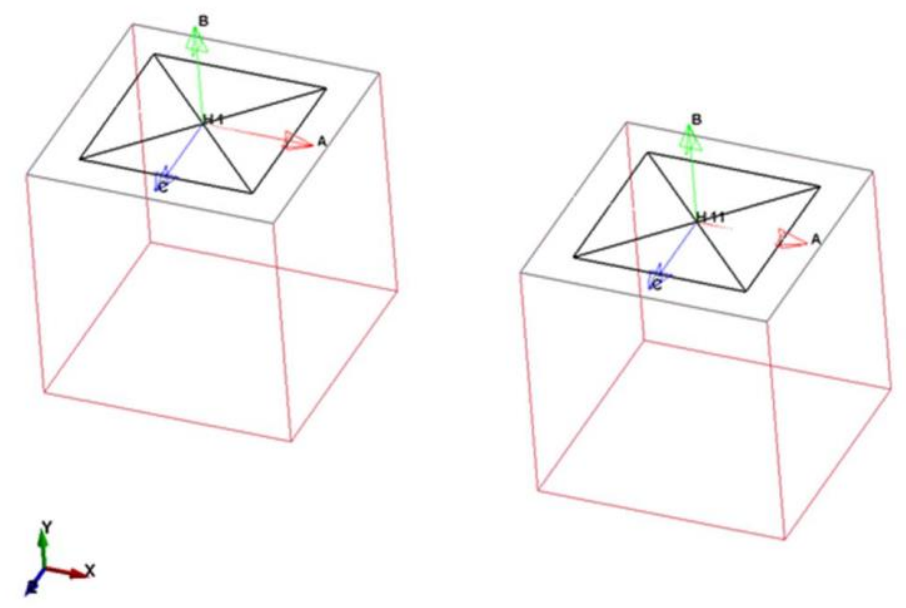

Figure 4. Single element models for normal loading verification analyses.

The results for the single element verification studies conducted for normal loading are shown in Figures 5-7, where Figure 5 shows the results for longitudinal tension/compression loading, Figure 6 shows the results for in-plane transverse tension/compression loading and Figure 7 shows the results for through-thickness tension/compression loading. In all cases, the computed results are compared to the corresponding tabulated curves that were input into MAT 213. As can be seen in the figures, in all cases the computed results correspond very well to the input curves, indicating that the underlying equations are appropriately implemented into the code.

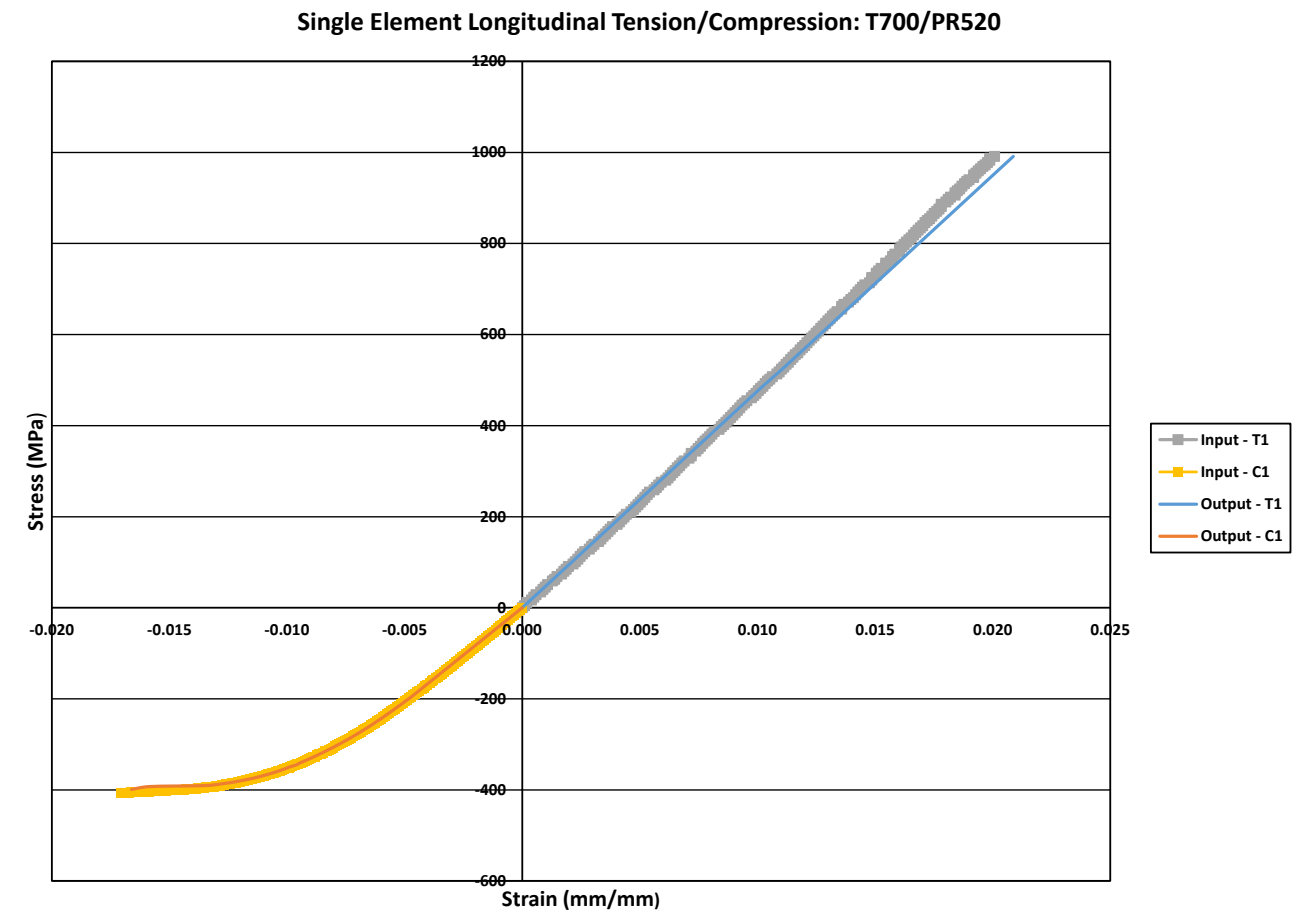

Figure 5. Single element longitudinal tension/compression analyses for T700/PR520 composite. 


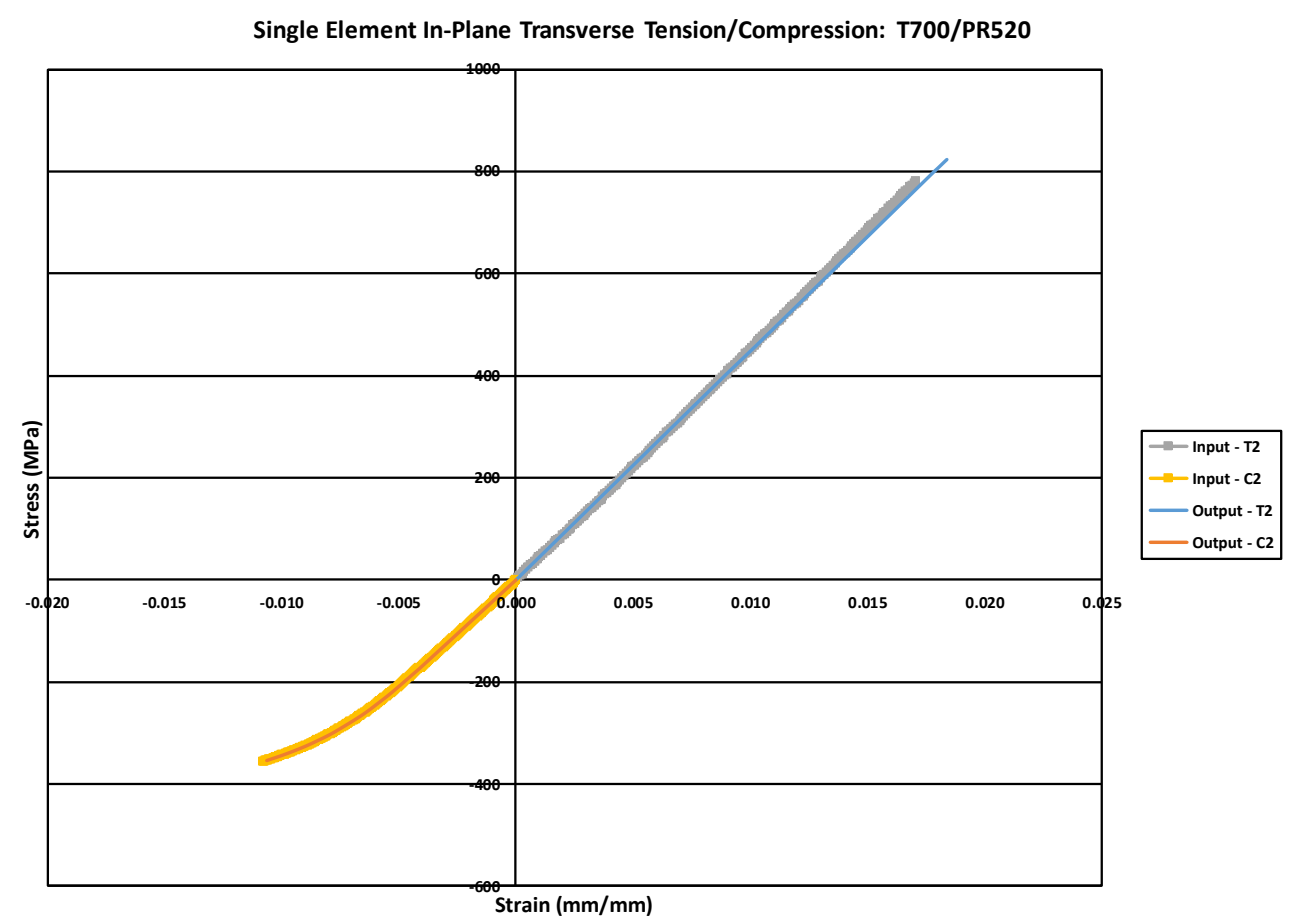

Figure 6. Single element in-plane transverse tension/compression analyses for T700/PR520 composite.

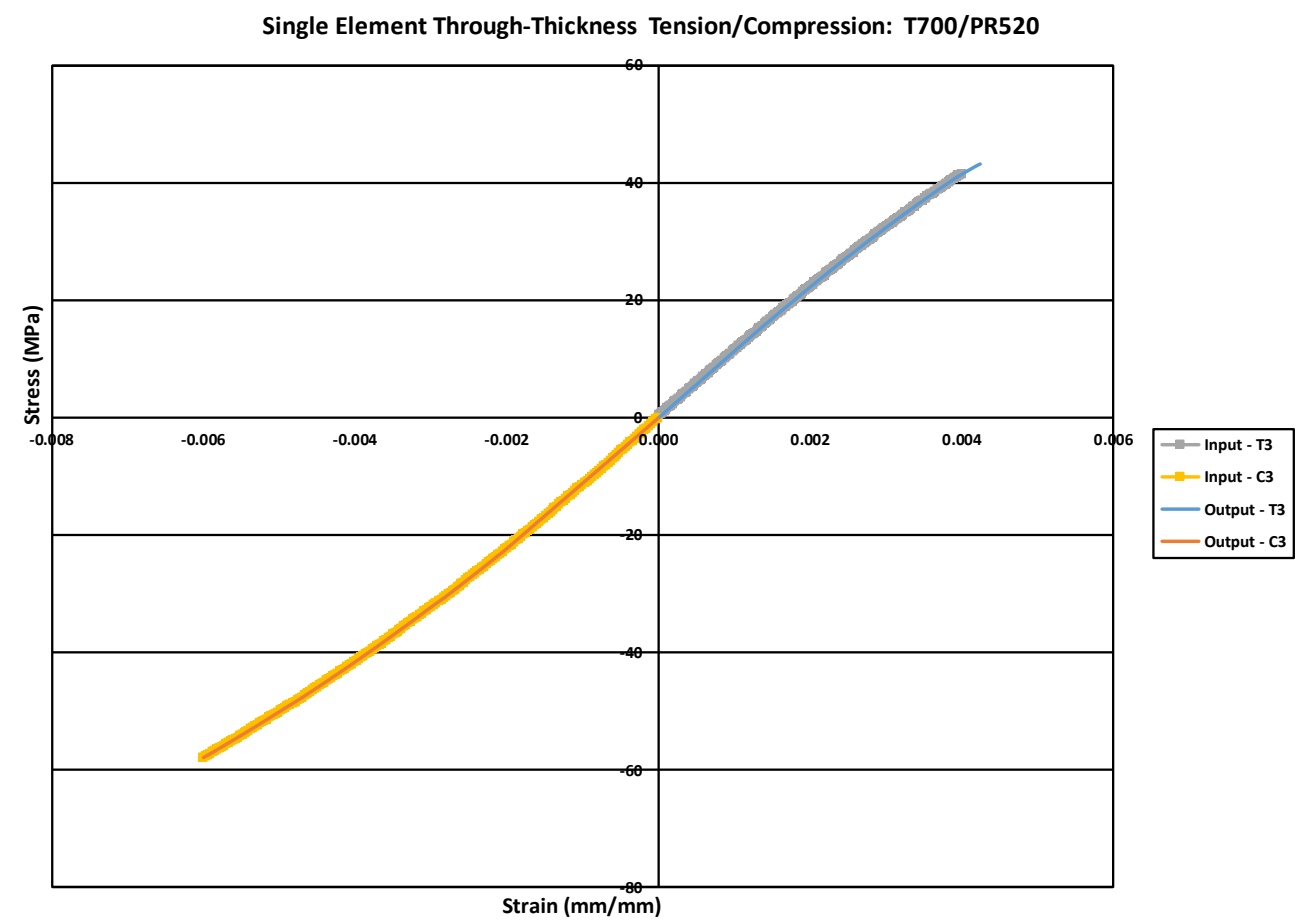

Figure 7. Single element through-thickness tension/compression analyses for T700/PR520 composite. 
To conduct the single element verification studies for shear loading, the models shown in Figure 8 were used. Specifically, in the single elements shown in Figure 8 the fiber directions are oriented $45^{\circ}$ to the indicated direction, i.e the red vector indicates the fiber direction in the model. Specifically, for the case of 1-2 shear the A and B vectors are in the XY plane, for the case of 2-3 shear the B and C vectors are in the XY plan and for the case of 31 shear the $\mathrm{A}$ and $\mathrm{C}$ vectors are in the $\mathrm{XY}$ plane. The cubes are loaded on two surfaces in the $\mathrm{XY}$ plane, one in compression and the other in tension, with the same magnitude of stress applied to provide a state of pure shear (zero mean stress). The notes of the unit cubes opposite the two loaded surfaces are constrained against normal displacement, the 24 other two degrees-of-freedom are also constrained at the node shared by the two constrained surfaces to prevent rigid body translation.
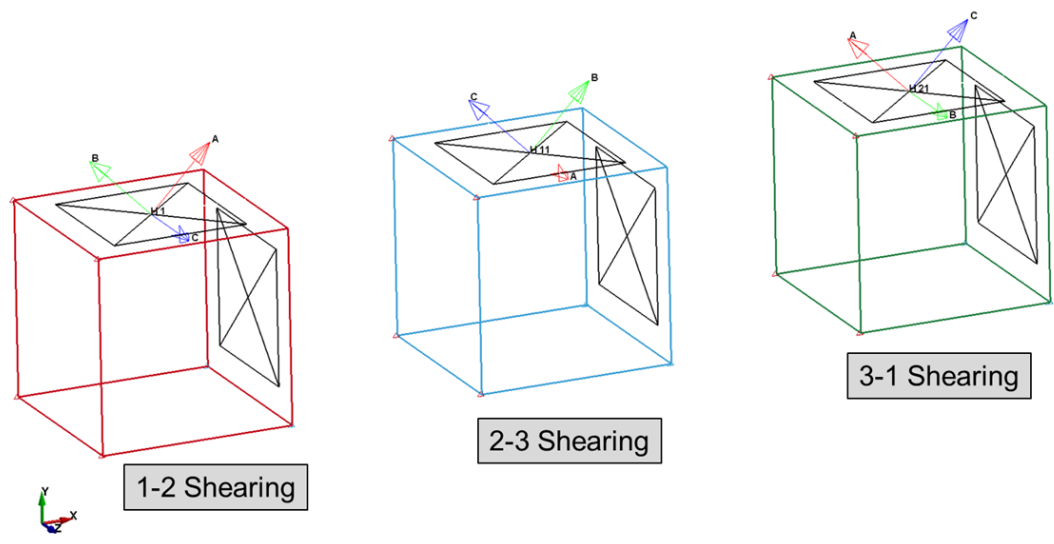

Figure 8. Single element models for shear loading verification analyses.

The results for the single element verification studies conducted for the three shear loading cases are shown in Figure 9. In all cases, once again the computed results are compared to the corresponding tabulated curves that were input into MAT 213. As can be seen in the figures, in all cases the computed results correspond very well to the input curves, indicating that the underlying equations are appropriately implemented into the code. 


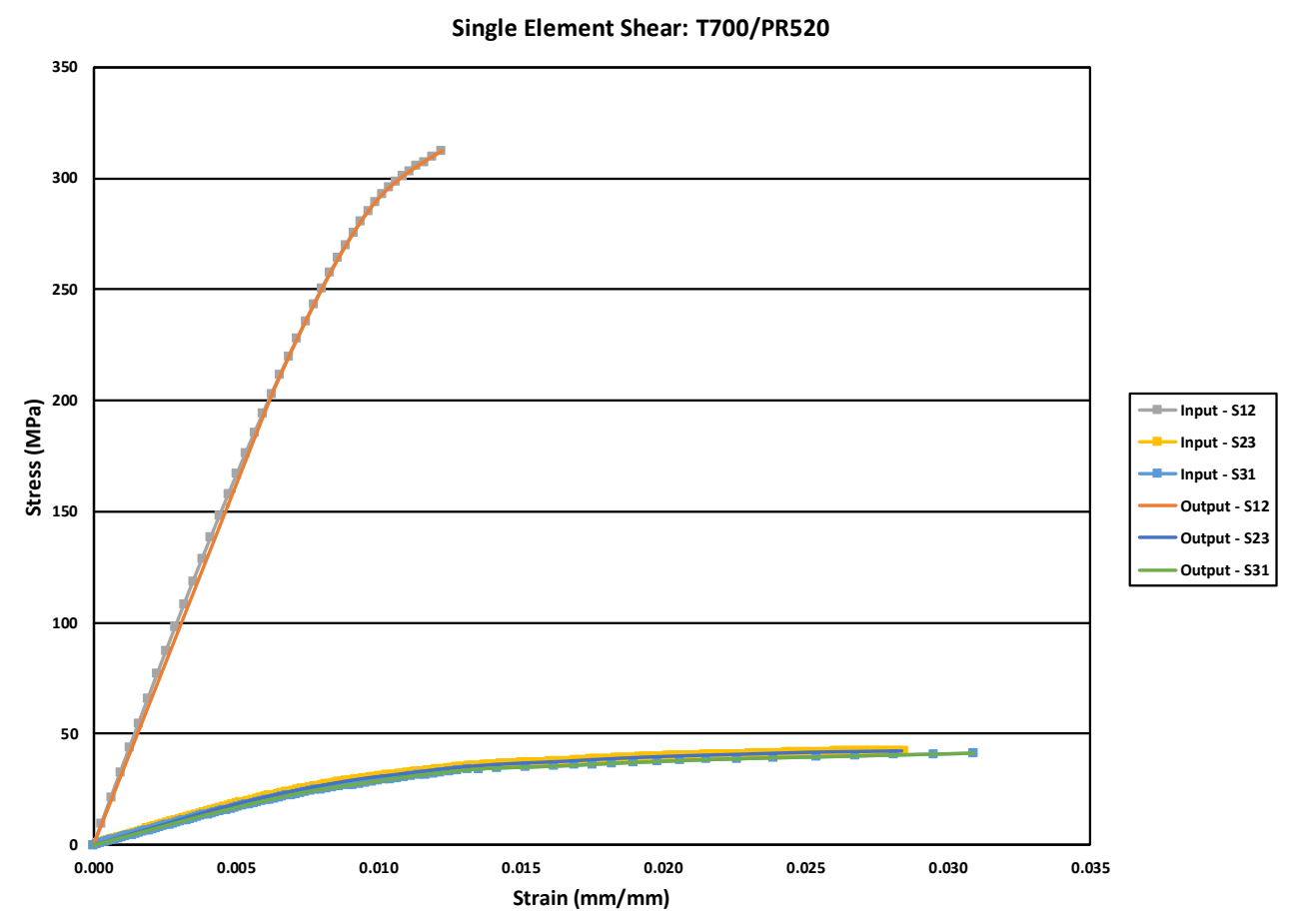

Figure 9. Single element shear analyses for T700/PR520 composite.

To examine the ability of MAT 213 to accurately analyze an impact event, flat panel impact tests conducted on a T800/F3900 laminated composite were simulated. Specifically, the composite is a T800S/3900-2B[P2352W-19] BMS8-276 Rev-H-Unitape fiber/resin unidirectional composite ${ }^{12}$. T800S is an intermediate modulus, high tensile strength graphite fiber. The epoxy resin system is labeled F3900 where a toughened epoxy is combined with small elastomeric particles to form a compliant interface or interleaf between fiber plies to resist impact damage and delamination ${ }^{13}$. The composite plate used in the impact tests had 16-plies (3.1 $\mathrm{mm}$ thick) and used a quasi-isotropic layup, [0/90/+45/-45] $]_{2}$. The dynamic impact test, conducted at NASA Glenn Research Center, was performed in accordance with ASTM D8101/D8101M-1 $7^{14}$, and consisted of an aluminum projectile striking the T800-F3900 composite plate. The mass of the projectile was $50 \mathrm{~g}$. The aluminum projectile was fired from a gas gun, impacting the panel with a velocity of $72 \mathrm{~m} / \mathrm{s}$. DIC equipment was used to obtain full field displacements with images captured using high speed cameras. The out-of-plane displacements at the center of the backside and the deformation patterns were chosen as comparison metrics. A convergence analysis and boundary condition study yielded a mesh where the composite parts were modeled with a total of 373,000 8-noded hexahedral elements with one-integration point. Each ply in the layup was modeled using one element through the thickness. A representative example of a panel that was used for testing is shown in Figure 10 and the finite element mesh used in the simulation is shown in Figure 11. The aluminum impactor used in the experiments is shown in Figure 12. 


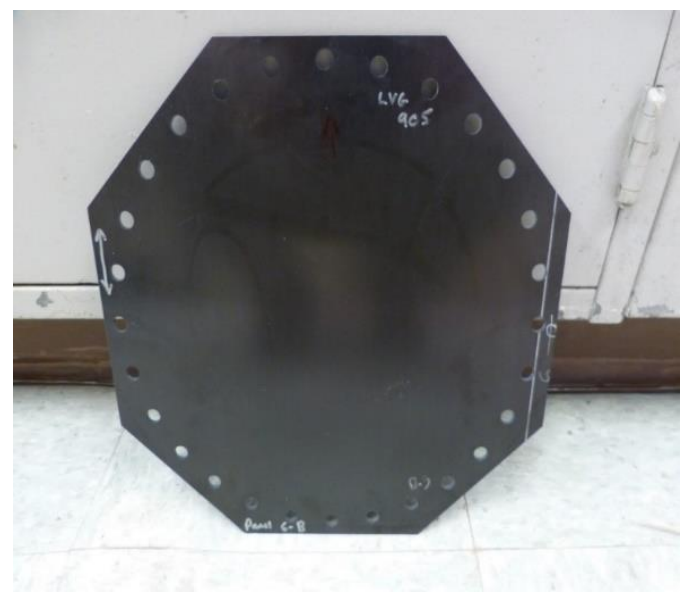

Figure 10. T800/F3900 panel used for impact testing.

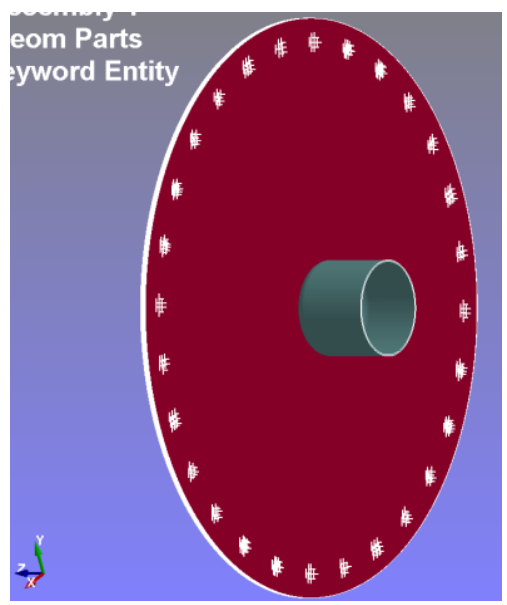

Figure 11. Finite element model used in simulations of T800/F3900 impact test.

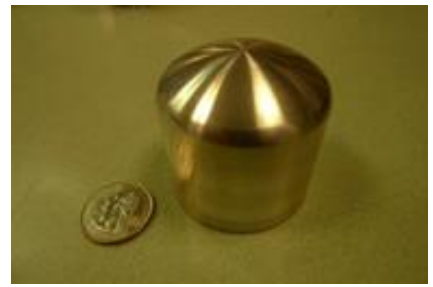

Figure 12. Aluminum impactor used in T800/F3900 impact tests.

Since MAT 213 does not include provisions for interlaminar delamination, cohesive zone elements were used to enhance the finite element model as significant delamination was observed during the experiment. A total of 350,000 cohesive zone elements, using a resident LS-DYNA cohesive zone material model, were modeled between each of the composite layers for a total of 15 layers of cohesive zone elements. The cohesive zone model utilized for this work employs a bilinear mixed mode traction-separation law to model delamination. The values used in the cohesive zone model were calibrated using publicly available experimental data for the T800-F3900 composite. a 
piecewise linear plasticity model with strain rate effects was used to model the aluminum impactor. The impactor was modeled using 17,000 8-noded hexahedral elements with one-integration point.

Simulations were conducted using only the deformation portion of the material model, in which all damage and failure effects were neglected. Another set of simulations were conducted where the effects of transverse and shear damage were incorporated into the material model. A plot of the backside displacement at the center of the composite panel as a function of time is shown in Figure 13. As can be seen in the figure, even the simulation with only the deformation model activated produced results that compared favorably to the experimental values, both qualitatively and quantitatively. Adding the damage capabilities to the model improved the qualitative comparison to the experimental results. Contour plots of the out-of-plane displacement patterns at the point of maximum displacement obtained experimentally are shown in Figure 14 and the corresponding results obtained numerically are shown in Figure 15. The contours in both plots represent identical levels of displacement. The numerically predicted displacement patterns qualitatively compare well with the experimental results.



Figure 13. Maximum backside displacement time history from T800/F3900 impact test and simulation.

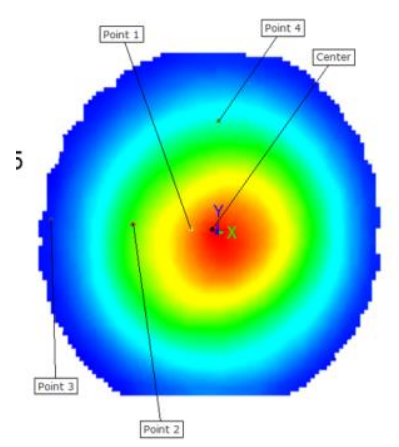

Figure 14. Backside displacement patterns observed in T800/F3900 impact test. 


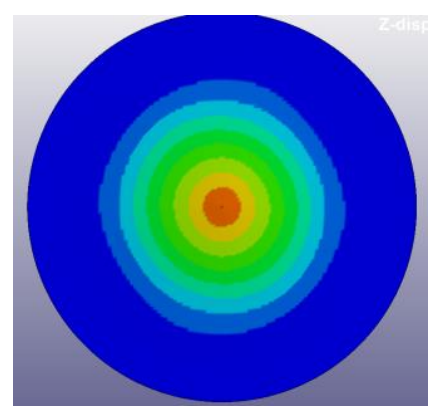

Figure 15. Predicted backside displacement patterns obtained from simulations of T800/F3900 impact test.

\section{Conclusions}

A generalized composite model suitable for use in polymer composite impact simulations has been developed. The model utilizes a plasticity based deformation model obtained by generalizing the Tsai-Wu failure criteria. A strain equivalent damage model has also been developed in which loading the material in a particular loading direction can lead to damage in multiple coordinate directions. A general, tabulated failure model has also been formulated. A systematic procedure for conducted verification and validation studies for the developed composite model has been developed. The procedure has been found to demonstrate the capability of the model for different materials composed of varying fiber architectures. Future efforts will involve continuing the $\mathrm{V} \& \mathrm{~V}$ studies for a wider range of material systems and composite architectures to further demonstrate the capability of the developed model to serve as a predictive tool for simulating impact events.

\section{Acknowledgments}

Authors Khaled, Shymasunder, and Rajan gratefully acknowledge the support of the Federal Aviation Administration through Grant \#12-G-001 entitled "Composite Material Model for Impact Analysis", William Emmerling, Technical Monitor and the National Aeronautics and Space Administration through Contract NN15CA32C, Robert Goldberg, Technical Monitor.

\section{References}

[1] LS-DYNA, Version 970. Livermore Software Technology Corporation, Livermore, CA, 2013.

[2] Matzenmiller, A., Lubliner, J, and Taylor, R.L., "A constitutive model for anisotropic damage in fiber-composites," Mechanics of Materials, Vol. 20, 1995, pp. 125-152.

[3] Sun, C.T., and Chen, J.L., "A Simple Flow Rule for Characterizing Nonlinear Behavior of Fiber Composites," Journal of Composite Materials, Vol. 23, 1989, pp. 1009-1020.

[4] Goldberg, R., Carney, K., DuBois, P., Hoffarth, C., Harrington, J., Rajan, S., and Blankenhorn, G, “Theoretical Development of an Orthotropic Elasto-Plastic Generalized Composite Model,” NASA/TM-2014-218347, 2014.

[5] Goldberg, R., Carney, K., DuBois, P., Hoffarth, C., Rajan, S., and Blankenhorn, G: "Incorporation of Plasticity and Damage Into an Orthrotropic Three-Dimensional Model With Tabulated Input Suitable for Use in Composite Impact Problems," NASA/TM-2015-218849, 2015.

[6] Daniel, I.M, and Ishai, O., Engineering Mechanics of Composite Materials. Oxford University Press, New York, 2006.

[7] Goldberg, R., Carney, K., DuBois, P., Hoffarth, C., Khaled, B., Shyamsunder, L., Rajan, S.; and Blankenhorn, G: "Incorporation of Failure Into an Orthotropic Three-Dimensional Model with Tabulated Input Suitable for Use in Composite Impact Problems," NASA/TM-2017-219492, 2017.

[8] Khan, A.S., and Huang, S., Continuum Theory of Plasticity. John Wiley and Sons, New York, 1995.

[9] Barbero, E.J., Finite Element Analysis of Composite Materials Using ABAQUS. CRC Press, Boca Raton, FL, 2013.

[10] Lemaitre, J., and Desmorat, R., Engineering Damage Mechanics: Ductile, Creep and Brittle Failures. Springer, Berlin, 2005.

[11] Fleischer M., Borrvall, T., and Bletzinger, K.-U., "Experience from using recently implemented enhancements for Material 36 in LS-DYNA 971 performing a virtual tensile test", 6th European LS-DYNA Users Conference, Gothenburg, Sweden, 2007.

[12] Raju K,S., and Acosta J.F. "Crashworthiness of composite fuselage structures -material dynamic properties, phase I," DOT/FAA/AR-09/8, 2010.

[13] Smith D.L., and Dow M.B., "Properties of three graphite/toughened resin composites," NASA Technical Paper $3102,1991$.

[14] ASTM D8101/D8101M-17, "Standard test method for measuring the penetration resistance of composite materials to impact by a blunt projectile," 2017. 